\title{
An Extremal Doubly Even Self-Dual Code of Length 112
}

\author{
Masaaki Harada \\ Department of Mathematical Sciences \\ Yamagata University \\ Yamagata 990-8560, Japan \\ mharada@sci.kj.yamagata-u.ac.jp
}

Submitted: Dec 29, 2007; Accepted: Aug 24, 2008; Published: Aug 31, 2008

Mathematics Subject Classifications: 94B05

\section{Dedicated to Professor Tatsuro Ito on His 60th Birthday}

\begin{abstract}
In this note, an extremal doubly even self-dual code of length 112 is constructed for the first time. This length is the smallest length for which no extremal doubly even self-dual code of length $n \not \equiv 0(\bmod 24)$ has been constructed.
\end{abstract}

\section{Introduction}

As described in [10], self-dual codes are an important class of linear codes for both theoretical and practical reasons. It is a fundamental problem to classify self-dual codes of modest length and determine the largest minimum weight among self-dual codes of that length. By the Gleason-Pierce theorem, there are nontrivial divisible self-dual codes over $\mathbb{F}_{q}$ for $q=2,3$ and 4 only, where $\mathbb{F}_{q}$ denotes the finite field of order $q$, and this is one of the reasons why much work has been done concerning self-dual codes over these fields.

A binary self-dual code $C$ of length $n$ is a code over $\mathbb{F}_{2}$ satisfying $C=C^{\perp}$ where the dual code $C^{\perp}$ of $C$ is defined as $C^{\perp}=\left\{x \in \mathbb{F}_{2}^{n} \mid x \cdot y=0\right.$ for all $\left.y \in C\right\}$ under the standard inner product $x \cdot y$. A self-dual code $C$ is doubly even if all codewords of $C$ have weight divisible by four, and singly even if there is at least one codeword of weight $\equiv 2(\bmod 4)$. Note that a doubly even self-dual code of length $n$ exists if and only if $n$ is divisible by eight. It was shown in [8] that the minimum weight $d$ of a doubly even self-dual code of length $n$ is bounded by $d \leq 4[n / 24]+4$. A doubly even self-dual code meeting this upper bound is called extremal.

The existence of extremal doubly even self-dual codes is known for the following lengths

$$
n=8,16,24,32,40,48,56,64,80,88,104,136
$$


and their existence was already known some 25 years ago (see [7, Fig. 19.2], [10, p. 273], see also [9] for length 64). We remark that the existence of an extremal doubly even self-dual code of length 72 is a long-standing open question [11] (see [10, Section 12]). 112 is the smallest length for which no extremal doubly even self-dual code of length $n \not \equiv 0$ (mod 24) has been constructed.

In this note, an extremal doubly even self-dual $[112,56,20]$ code is constructed for the first time. Moreover, this code has a larger minimum weight than the previously known linear $[112,56]$ codes. For length $n=110,112$, singly even self-dual codes with minimum weight 18 are also constructed using the extremal doubly even self-dual code of length 112. These codes have larger minimum weights than the previously known self-dual codes of that length.

\section{An Extremal Doubly Even Self-Dual Code of Length 112}

Let $A, B$ be the $28 \times 28$ circulant matrices with first rows $r_{A}, r_{B}$, respectively, where

$$
\begin{aligned}
& r_{A}=(1,0,0,1,0,1,0,0,0,0,0,0,0,0,1,0,1,0,0,1,1,1,1,0,0,1,1,1), \\
& r_{B}=(1,0,1,1,1,0,0,1,1,1,1,1,0,0,0,0,1,1,1,1,0,1,1,1,1,0,1,1) .
\end{aligned}
$$

Let $C_{112}$ be the code with generator matrix

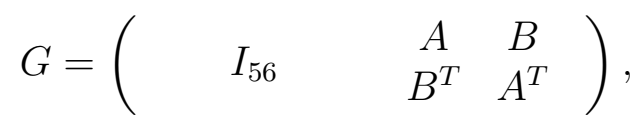

where $I_{n}$ denotes the identity matrix of order $n$ and $A^{T}$ is the transposed matrix of $A$. Since $A B=B A, A A^{T}+B B^{T}=I_{28}$ and the sum of the weights of $r_{A}$ and $r_{B}$ is $31, C_{112}$ is a doubly even self-dual code (see [6] for the construction method). If $C_{112}$ contains a codeword $c$ of weight $\leq 16$ then $c$ can be expressed as a sum of at most eight rows of $G$ or a sum of at most seven rows of a parity-check matrix

$$
H=\left(\begin{array}{ccc}
A^{T} & B & I_{56} \\
B^{T} & A &
\end{array}\right),
$$

since $C_{112}$ is self-dual. We have verified that the weights of the sums of all $g$ rows of $G$ and the sums of $h$ rows of $H$ are greater than or equal to 20 for $g=1,2, \ldots, 8$ and $h=1,2, \ldots, 7$. This shows that $C_{112}$ has minimum weight 20 (the minimum weight is also verified by Magma [1]). Therefore $C_{112}$ is an extremal doubly even self-dual code and we have the following:

Theorem 1. There is an extremal doubly even self-dual code of length 112.

The code $C_{112}$ has a larger minimum weight than not only the previously known selfdual codes of length 112 but also the previously known linear [112,56] codes (see [2] and $[5])$. 
The weight enumerator of an extremal doubly even self-dual code is given in [8] for lengths $n \leq 200$. We have verified that $C_{112}$ is generated by the codewords of minimum weight. In addition, we have verified by MAGMA that $C_{112}$ has automorphism group $\operatorname{Aut}\left(C_{112}\right)$ of order 112 which acts transitively on the coordinates. A generator matrix of $C_{112}$ and programs written in MAGMA to verify the above properties can be obtained electronically from http://sci.kj.yamagata-u.ac.jp/ ${ }^{\sim m h a r a d a / P a p e r / 112 . m a g m a . ~}$

Now the smallest length for which no extremal doubly even self-dual code of length $n \not \equiv 0(\bmod 24)$ is known is 128 and the largest length for which an extremal doubly even self-dual code is known is 136 .

\section{$3 \quad$ Related Singly Even Self-Dual Codes}

The minimum weight $d$ of a singly even self-dual code of length $n$ is bounded by $d \leq$ $4[n / 24]+4$, unless $n \equiv 22(\bmod 24)$ when $d \leq 4[n / 24]+6$ or $n \equiv 0(\bmod 24)$ when $d \leq 4[n / 24]+2$ (see [10]). A singly even self-dual code meeting this upper bound is called extremal.

\subsection{Length 110}

Let $S_{C_{112}}(i, j)$ be the code obtained by subtracting two coordinates $i, j$ (i.e., taking all codewords with $(0,0),(1,1)$ in the coordinates and deleting the coordinates) from $C_{112}$. The codes $S_{C_{112}}(i, j)$ are self-dual codes of length 110 and minimum weight 18 or 20 .

Let $M=\left(m_{i j}\right)$ be the $355740 \times 112$ matrix with rows composed of the codewords of weight 20 in $C_{112}$. Let $n_{11}^{(j)}$ and $n_{00}^{(j)}$ be the numbers of integers $r(1 \leq r \leq 355740)$ with $m_{r 1}=m_{r j}=1$ and $m_{r 1}=m_{r j}=0$, respectively, for $j(2 \leq j \leq 112)$. It is enough to consider only the case $i=1$ since $\operatorname{Aut}\left(C_{112}\right)$ acts transitively on the coordinates. We have verified that $n_{11}^{(j)}$ are positive for all $j(2 \leq j \leq 112)$. Hence the codes $S_{C_{112}}(i, j)$ obtained by subtracting all pairs of two coordinates have minimum weight 18 , that is, these self-dual codes are non-extremal. However, these self-dual $[110,55,18]$ codes have larger minimum weights than the previously known self-dual codes of length 110 (see [4, Table 2]). By comparing $n_{11}^{(j)}$ and $n_{00}^{(j)}$ for all $j$, it turns out that over 50 self-dual $[110,55,18]$ codes obtained by subtracting have different weight enumerators.

\section{$3.2 \quad$ Length 112}

Recall that two self-dual codes $C$ and $C^{\prime}$ of length $n$ are called neighbors if the dimension of $C \cap C^{\prime}$ is $n / 2-1$. Let $v \in \mathbb{F}_{2}^{112}$ be a vector of weight 4 . Then

$$
N_{C_{112}}(v)=\left(C_{112} \cap\langle v\rangle^{\perp}\right) \cup\left\{u+v \mid u \in\left(C_{112} \backslash\left(C_{112} \cap\langle v\rangle^{\perp}\right)\right)\right\}
$$

is a singly even self-dual neighbor of $C_{112}$ with minimum weight 18 or 20 (see [3] for the construction method). 
Let $M=\left(m_{i j}\right)$ be the $355740 \times 112$ matrix as above. We denote the support of $v$ by $\operatorname{supp}(v)=\left\{i_{1}, i_{2}, i_{3}, i_{4}\right\}$. Let $n_{j}^{(v)}$ be the number of integers $r(1 \leq r \leq 355740)$ with

$$
\mathrm{wt}\left(m_{r i_{1}}, m_{r i_{2}}, m_{r i_{3}}, m_{r i_{4}}\right)=j(j=0,1,2,3,4),
$$

where $\operatorname{wt}(x)$ denotes the weight of a vector $x$. From the construction, the numbers of

codewords of weights 18 and 20 in $N_{C_{112}}(v)$ are given by $n_{3}^{(v)}$ and $n_{0}^{(v)}+n_{2}^{(v)}+n_{4}^{(v)}$, respectively. We have verified that $n_{3}^{(v)}$ are positive for all $v$ with $\operatorname{supp}(v)=\left\{1, i_{2}, i_{3}, i_{4}\right\}$. Hence the codes $N_{C_{112}}(v)$ have minimum weight 18, that is, these codes are non-extremal. However, these singly even self-dual $[112,56,18]$ codes have larger minimum weights than the previously known singly even self-dual codes of length 112 (see [4, Table 2]). By comparing $n_{3}^{(v)}$ and $n_{0}^{(v)}+n_{2}^{(v)}+n_{4}^{(v)}$ for all $v$ with $\operatorname{supp}(v)=\left\{1,2, i_{3}, i_{4}\right\}$, it turns out that over 100 singly even self-dual $[112,56,18]$ neighbors $N_{C_{112}}(v)$ have different weight enumerators.

For lengths $n=110,112$, singly even self-dual codes with minimum weight 18 have been constructed. Hence the largest minimum weight among singly even self-dual codes of length $n$ is 18 or 20 .

Acknowledgment. The author would like to thank T. Aaron Gulliver and Radinka Yorgova for useful conversations.

\section{References}

[1] W. Bosma and J. Cannon, Handbook of Magma Functions, Available online at "http://magma.maths . usyd.edu . au/magma/".

[2] A.E. Brouwer, "Bounds on the size of linear codes," in Handbook of Coding Theory, V.S. Pless and W.C. Huffman (Editors), Elsevier, Amsterdam 1998, pp. 295-461.

[3] R. Brualdi and V. Pless, Weight enumerators of self-dual codes, IEEE Trans. Inform. Theory 37 (1991), 1222-1225.

[4] P. Gaborit and A. Otmani, Experimental constructions of self-dual codes, Finite Fields Appl. 9 (2003), 372-394.

[5] M. Grassl, Code tables: Bounds on the parameters of various types of codes, Available online at "http://www. codetables.de/".

[6] M. Harada, W. Holzmann, H. Kharaghani and M. Khorvash, Extremal ternary selfdual codes constructed from negacirculant matrices, Graphs Combin. 23 (2007), 401-417.

[7] F.J. MacWilliams and N.J.A. Sloane, "The Theory of Error-Correcting Codes," North-Holland, Amsterdam, 1977.

[8] C.L. Mallows and N.J.A. Sloane, An upper bound for self-dual codes, Inform. Control 22 (1973), 188-200. 
[9] G. Pasquier, A binary extremal doubly even self-dual code $(64,32,12)$ obtained from an extended Reed-Solomon code over $F_{16}$, IEEE Trans. Inform. Theory 27 (1981), 807-808.

[10] E. Rains and N.J.A. Sloane, "Self-dual codes," in Handbook of Coding Theory, V.S. Pless and W.C. Huffman (Editors), Elsevier, Amsterdam, 1998, pp. 177-294.

[11] N.J.A. Sloane, Is there a $(72,36) d=16$ self-dual code? IEEE Trans. Inform. Theory 19 (1973), 251. 